\title{
MACDONALD'S CONSTANT TERM CONJECTURES FOR EXCEPTIONAL ROOT SYSTEMS
}

\author{
FRANK G. GARVAN AND GASTON GONNET
}

\begin{abstract}
We announce proofs of Macdonald's constant term conjectures for the affine root systems $S\left(F_{4}\right)$ and $S\left(F_{4}\right)^{\nu}$. We also give an algorithm for deciding the conjectures for the remaining root systems $S\left(E_{6}\right), S\left(E_{7}\right)$, and $S\left(E_{8}\right)$ and prove that the constant term in question can indeed be expressed in closed form. Combined with previous work of ZeilbergerBressoud, Kadell, and Gustafson, our results imply that Macdonald's conjectures are true in form for any root system, and the complete truth of Macdonald's conjectures is a finite number of mips away.
\end{abstract}

\section{INTRODUCTION AND RESULTS}

Root systems and reflection groups occupy a central position in Lie theory [Hu1], finite groups [C] and other branches of mathematics, and are also an intriguing object of study for their own sake [Hu2, B]. In 1972, Macdonald [Ma1] proved a series of "formal" identities, one for every affine root system, that for the simplest affine root system $S\left(A_{1}\right)$ specialized to Jacobi's triple product formula. These formulas had numerous number-theoretic applications [Ma1, D] and also constituted the "tip of the iceberg" that led Victor Kac to the theory of representations of Kac-Moody algebras [Kac, pp. xiii, xiv]. In 1982, Macdonald [Ma2] conjectured a collection of constant term identities that constituted "finite forms" generalizations of his celebrated identities. The most general of these conjectures, which was obtained by jointly generalizing an earlier conjecture of his (qM) and a conjecture of Morris [Mo] (see also [A]) for $G_{2}$, has the form

C. T. $\prod\left(x^{\alpha} ; q^{u_{\alpha}}\right)_{k_{\alpha}}\left(q^{u_{\alpha}} x^{-\alpha} ; q^{u_{\alpha}}\right)_{k_{\alpha}}=$ a certain explicit product.

Received by the editors November 1, 1990 and, in revised form, November 21, 1990.

1980 Mathematics Subject Classification (1985 Revision). Primary 05A30, 33A35, 17B20; Secondary 17B67. 
Here C.T. means constant term in the Laurent polynomial in the $x^{ \pm \alpha} ; R$ is the underlying finite root system, $k_{\alpha}$ are nonnegative integers satisfying $k_{\alpha}=k_{\beta}$ whenever $|\alpha|=|\beta| ; u_{\alpha}$ are certain constant integers associated with affine root system [Ma1] and $(a ; q)_{k}$ is the standard $q$-notation

$$
(a ; q)_{k}:=(1-a)(1-a q) \cdots\left(1-a q^{k-1}\right) .
$$

The "certain explicit product" that appears on the right side of (qM-M) can be looked up in [Ma2]. Since every affine root system is a direct sum of irreducible ones [Ma1, $\mathrm{Kac}, \mathrm{Hu} 2]$, it suffices to prove the conjecture for the latter. Recently, Gustafson [Gu] completed the proof of Macdonald's conjecture for all the infinite families by proving it for the infinite families $S\left(C_{n}\right), S\left(B_{n}\right)^{\nu}, S\left(C_{n}\right)^{\nu}$. The other infinite families were done previously by Zeilberger and Bressoud [Z-B] $\left(S\left(A_{n}\right)\right)$ and Kadell [Kad] $\left(S\left(B_{n}\right), S\left(D_{n}\right), S\left(B C_{n}\right)\right)$. In addition it is known for the exceptional root systems $S\left(G_{2}\right)$ [Ha, Z1], and $S\left(G_{2}\right)^{\nu}$ [Z2]. It thus remains open for $S\left(F_{4}\right)$, $S\left(F_{4}\right)^{\nu}, S\left(E_{6}\right), S\left(E_{7}\right)$, and $S\left(E_{8}\right)$. For the special case $q=1$, it was proved for $F_{4}$ [Ga], and for all root systems by Opdam [O]. Here we announce the truth of Macdonald's conjecture for the affine root systems $S\left(F_{4}\right)$ and $S\left(F_{4}\right)^{\nu}$. We also prove that (qM$\mathrm{M})$ is true in form for the remaining affine root systems $S\left(E_{6,7,8}\right)$. In other words, we show that the left side of (qM-M) can indeed be expressed as a certain finite product, although we are unable to say whether it is the one conjectured by Macdonald. Furthermore, we give an effective algorithm for finding this out, and the only obstacle for knowing for sure is our limited computing resources. Since every affine root system is a direct sum of irreducible ones, it follows that our results, combined with the previous work on the infinite families, implies that Macdonald's conjecture is true in form for every affine root system, and there exists an effective algorithm for settling it in full.

\section{SKETCH OF THE PROOF}

The proof is an enhancement of Zeilberger's method [Z2]. Zeilberger was inspired, in turn, by earlier work of $\mathrm{D}$. Stanton [Sta] and J. Stembridge [Ste]. For the sake of simplicity let us take $k_{\alpha} \equiv k$, and the affine root system to be of the form $S(R)$. The proof for the general case follows the same ideas but is more involved. For 
this case, (qM-M) becomes

$$
\text { C. T. } \prod_{\alpha \in R^{+}}\left(x^{\alpha}\right)_{k}\left(q x^{-\alpha}\right)_{k}=\left[\begin{array}{c}
d_{1} k \\
k
\end{array}\right] \ldots\left[\begin{array}{c}
d_{l} k \\
k
\end{array}\right],
$$

where $d_{1}, \ldots, d_{l}$ are the fundamental invariants of the root system $R$, and $\left[\begin{array}{l}a \\ b\end{array}\right]$ is the $q$-binomial coefficient. The first step is to use Stembridge's idea [Ste] and consider the antisymmetric version, that can be shown to be equivalent:

$$
\begin{aligned}
\text { C. T. } & \prod_{\alpha \in R^{+}}\left(x^{\alpha}\right)_{k}\left(q x^{-\alpha}\right)_{k-1} \\
= & \frac{\left(1-q^{k}\right)^{l}}{\left(1-q^{k d_{1}}\right) \cdots\left(1-q^{k d_{l}}\right)}\left[\begin{array}{c}
d_{1} k \\
k
\end{array}\right] \ldots\left[\begin{array}{c}
d_{l} k \\
k
\end{array}\right] .
\end{aligned}
$$

Denote the product on the left by $F_{k}$, and the whole left side by $H_{k}$ (so $H_{k}=$ C. T. $F_{k}$ ). By using

$$
\left(x^{\alpha}\right)_{k+1}\left(q x^{-\alpha}\right)_{k}=\left(1-q^{k} x^{\alpha}\right)\left(1-q^{k} x^{-\alpha}\right)\left(x^{\alpha}\right)_{k}\left(q x^{-\alpha}\right)_{k-1} \text {, }
$$

for every $\alpha$, expanding, and using the antisymmetry with respect to the natural action of the Weyl group, Zeilberger [Z2] expresses $H_{k+1}$ as a linear combination of $H_{k}$ and some "neighboring coefficients", with coefficients that are polynomials in $q^{k}$. Then he computes $F_{k}\left(x_{1} \rightarrow q x_{1}\right) / F_{k}$, expresses it as a rational function $P / Q$, cross multiplies, and multiplies by the monomial $x^{\beta}$ from the left. He then applies the functional C.T., to get homogeneous linear equations, with coefficients that are polynomials in $\left(q, q^{k}\right)$, relating the neighboring coefficients with each other and with $H_{k}$. If in luck, he gets enough independent equations to express all these neighboring coefficients as a product of a rational function in $\left(q^{k}, q\right)$ times $H_{k}$. He then substitutes all these expressions into the above mentioned expression for $H_{k+1}$, and, by dividing through by $H_{k}$, he gets an expression for $H_{k+1} / H_{k}$ as a sum of rational functions, and therefore as a rational function in $\left(q^{k}, q\right)$. Our enhancement of Zeilberger's method consists in proving that by a judicious change of basis, and by a systematic choice of monomials $x^{\beta}$, it is possible to guarantee that Zeilberger's method always works: The system of linear equations can be made triangular, and it involves all the necessary neighboring coefficients. In particular it follows that $H_{k+1} / H_{k}$ is always a rational function, and thus the constant term has always closed form. Similar considerations apply to the more general case of conjecture (qM-M). 
The method was completely implemented for $S\left(F_{4}\right)$ and $S\left(F_{4}\right)^{\nu}$, using MAPLE. Full proofs will appear elsewhere [G-G].

\section{ACKNOWLEDGMENTS}

I would also like to thank Dennis Stanton for many helpful discussions.

\section{REFERENCES}

[A] Richard Askey, Integration and computers, The Proceedings of the Computer Algebra Conference (D. and G. Chudnovsky, eds.) (to appear).

[B] N. Bourbaki, Groupes et algèbres de Lie, (Chaps. 4, 5, and 6), Hermann, Paris, 1968.

[C] R. Carter, Simple groups of Lie type, Wiley, London, 1972.

[D] F. Dyson, Missed opportunities, Bull. Amer. Math. Soc. (N.S.) 78 (1972), 635-653.

[Ga] Frank Garvan, A proof of the Macdonald-Morris root system conjectures for $F_{4}$, SIAM J. Math. Anal. 21 (1990), 803-821.

[G-G] Frank Garvan and Gaston Gonnet, $A$ proof of the two parameter q-case of the Macdonald-Morris constant term root system conjecture for $S\left(F_{4}\right)$ and $S\left(F_{4}\right)^{\nu}$ via Zeilberger's method, preprint.

[Gu] Robert A. Gustafson, A generalization of Selberg's beta integral, Bull. Amer. Math. Soc. (N.S.) 22 (1990), 97-105.

[Ha] Laurent Habsieger, La q-conjecture de Macdonald-Morris pour $G_{2}$, C. R. Acad. Sci. Paris Sér. I Math. 303 (1986), 211-213.

[Hu1] J. E. Humphreys, Introduction to Lie algebras and representation theory, Springer-Verlag, New York, 1972.

[Hu2] _ Reflection groups and Coxeter groups, Cambridge University Press, Cambridge, 1990.

[Kac] Victor Kac, Infinite dimensional Lie algebras, (2nd ed.), Cambridge University Press, Cambridge, 1985.

[Kad] Kevin Kadell, $A$ proof of the q-Macdonald-Morris conjecture for $B C_{n}$, preprint.

[Ma1] Ian G. Macdonald, Affine root systems and Dedekind's $\eta$-function, Invent. Math. 15 (1972), 91-143.

[Ma2] _ Some conjectures for root systems, SIAM J. Math. Anal. 13 (1982), 988-1007.

[Mo] Walter G. Morris, II, Constant term identities for finite and affine root systems, Ph.D. thesis, Univ. of Wisconsin-Madison, 1982.

[O] Erik Opdam, Some applications of hypergeometric shift operators, Invent. Math. 98 (1989), 1-18.

[Sta] Dennis Stanton, Sign variations of the Macdonald identities, SIAM J. Math. Anal. 17 (1986), 1454-1460.

[Ste] John Stembridge, $A$ short proof of Macdonald's conjecture for the root systems of type A, Proc. Amer. Math. Soc. 102 (1988), 777-786.

[Z1] Doron Zeilberger, $A$ proof of the $G_{2}$ case of Macdonald's root system-Dyson conjecture, SIAM J. Math. Anal. 18 (1987), 880-883. 
[Z2] - Unified approach to Macdonald's root system conjectures, SIAM J. Math. Anal. 19 (1988), 987-1013.

[Z-B] Doron Zeilberger and David Bressoud, A proof of Andrews' q-Dyson conjecture, Discrete Math. 54 (1985), 201-224.

Department of Mathematics, University of Florida, Gainesville, FLORIDA 32611

Current address: Department of Mathematics, Statistics, and Computing Science, Dalhousie University, Halifax, Nova Scotia, Canada, B3H 3J5

E-mail address: frank@cs.dal.ca, frank@math.ufl.edu

INFORMATIK, ETH, ZURICH, SWITZERLAND 8092

E-mail address: gonnet@inf.ethz.ch 
